\title{
ERL R\&D: Laser and Laser Light Transport
}

\author{
Brian Sheehy
}

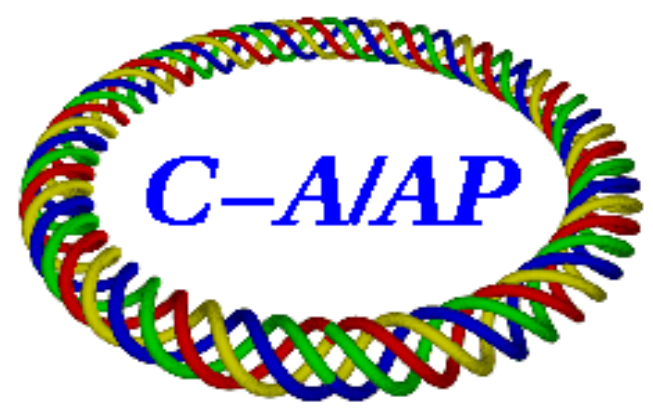

\section{Collider-Accelerator Department Brookhaven National Laboratory \\ Upton, NY 11973}

Notice: This document has been authorized by employees of Brookhaven Science Associates, LLC under Contract No. DE-AC02-98CH10886 with the U.S. Department of Energy. The United States Government retains a nonexclusive, paid-up, irrevocable, world-wide license to publish or reproduce the published form of this document, or allow others to do so, for United States Government purposes. 


\section{ERL R\&D: Laser and laser light transport}

Operation of the photocathode gun in the ERL requires that a tightly controlled optical pulse train, consisting of temporally and spatially shaped pulses, be delivered at the photocathode in synchrony with the RF field in the gun cavity. The pulse train must also be dynamically variable, in order to tune or ramp up the current in the ERL. A laser was developed especially for this task by Lumera Laser GmbH, of Kaiserslautern Germany, under design supervision and review of the ERL project. Following the final design review, the laser was delivered in August 2009. Preliminary tests certifying its compliance with design specifications have been performed, with further tests planned following the final certification of the ERL laser room in January 2010. The development of the necessary spatial and temporal shaping techniques is an ongoing project: proof of principle experiments have been successfully carried out with a laser of similar pulse width, operating at $532 \mathrm{~nm}$ and $81.5 \mathrm{MHz}$. The next stage is to evaluate the application of these techniques and alternatives, using the operations laser. A transport line has been designed and the propagation of a shaped pulse through it to the photocathode simulated and tested experimentally. As the performance of the complete photocathode drive system is critical for ERL operation, an extensive set of diagnostics will be in place to monitor and maintain its performance. The block diagram in Fig. 1 breaks the optical system down into its basic components, which are discussed in turn in the following sections.

\section{Laser Requirements}

The performance requirements of the laser are tabulated in Table 1. The specified wavelength is $355 \mathrm{~nm}$, but the laser produces 3 wavelengths: 355,532 , and $1064 \mathrm{~nm}$. Operation is planned at $355 \mathrm{~nm}$, as the increase in the quantum efficiency of the photocathode relative to $532 \mathrm{~nm}$ is greater than the decrease in available pulse energy. $\mathrm{CsK}_{2} \mathrm{Sb}$ cathodes can operate at $532 \mathrm{~nm}$ however, and there is some interest in studying gun performance at that wavelength, since pulse generation and shaping is easier at that wavelength, and in possible future configurations (eg higher repetition rates), shorter wavelength sources might be more difficult to produce. The $532 \mathrm{~nm}$ and $1064 \mathrm{~nm}$ light is also useful for diagnostic applications.

The repetition rate of $9.38 \mathrm{MHz}$ is the $75^{\text {th }}$ subharmonic of the RF frequency of the gun and accelerating cavity, $703.5 \mathrm{MHz}$. Synchronization with the RF field in the gun is extremely important; asynchrony impacts beam energy fluctuations, emittance, energy recovery, and ultimately overall stability. The total jitter must be less than 1 psec rms. Timing requirements also include the ability to ramp up the repetition rate of the laser while maintaining synchronization, in order to run the ERL at low repetition rate while tuning up, and ramp up its current in operation.

The optimal width of the optical pulse at the photocathode is much longer than the 10-12 picoseconds specified for the laser. The pulse shape of the drive pulse is optimally flat- 


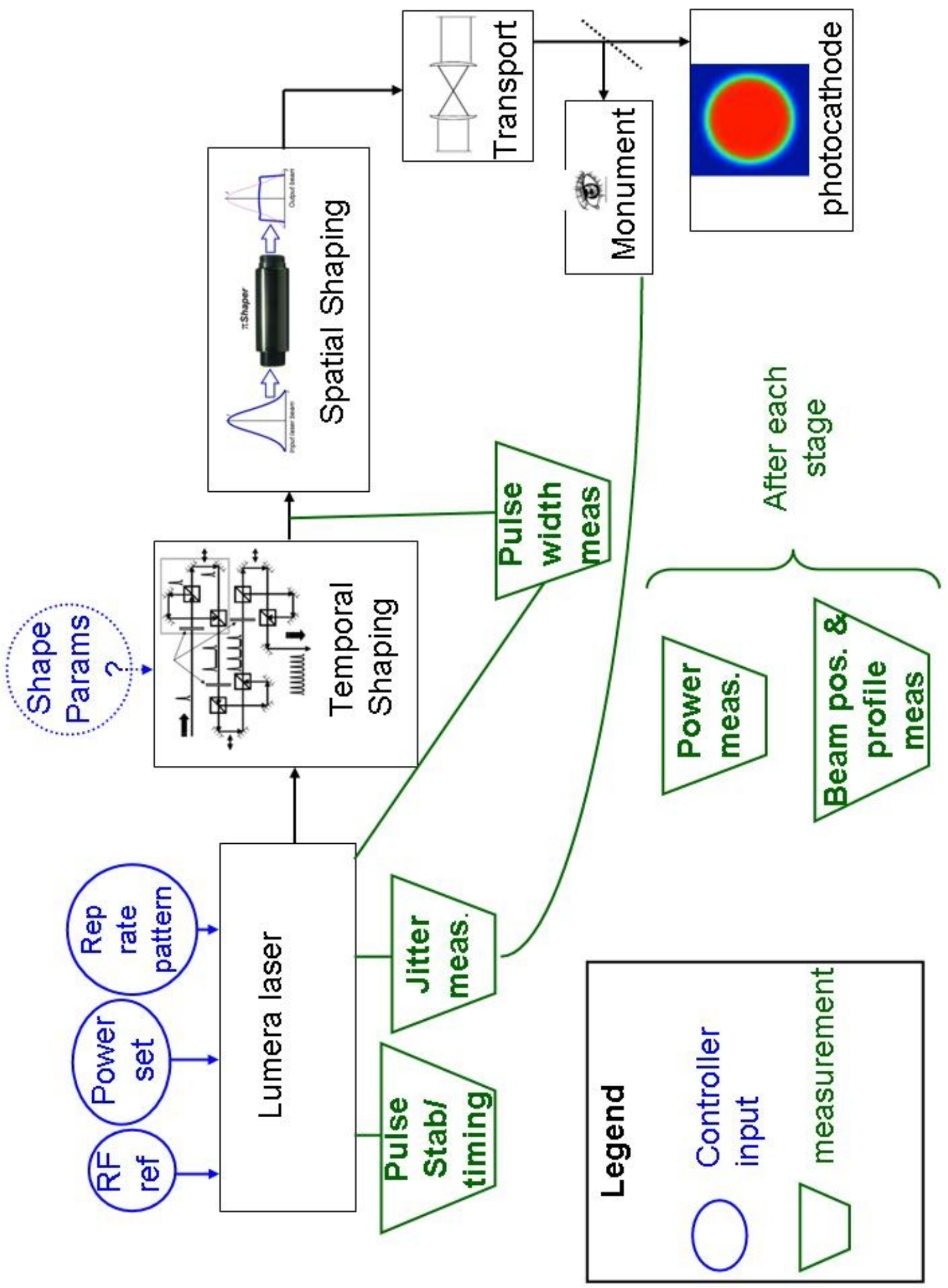

Figure 1 Schematic system overview 
topped, and the specified width for the nominally $\operatorname{sech}^{2}$-shaped pulse from the laser was chosen to obtain, within the constraints imposed by this type of mode-locked laser, an adequately short rise and fall time in the photocathode drive pulse produced by the shaping methods described below. Similarly, the mode quality specification is driven by the mode requirements of the spatial shaping techniques. The total power requirement of $5 \mathrm{~W}$ is dictated by the desired maximum ERL current of $50 \mathrm{~mA}$. This current would require $1.7 \mathrm{~W}$ of $355 \mathrm{~nm}$ light delivered at the photocathode, at the optimal quantum efficiency of $10 \%$, leaving over a three-watt margin to cover losses in shaping, transport, and diagnostics, and to compensate for less than optimal quantum efficiency.

\begin{tabular}{|c|c|}
\hline Master RF Repetition Rate & $703.5 \mathrm{MHz}$ \\
\hline Laser Pulse Reptition Frequency & $9.38 \mathrm{MHz}$ \\
\hline Frequency tunability & $+/-1 \mathrm{MHz}$ \\
\hline $\begin{array}{c}\text { Synchronization deviation to } \\
\text { master oscillator }\end{array}$ & $<1 \mathrm{ps}$ \\
\hline Pulse Length & $5-12 \mathrm{ps}$ \\
\hline Jitter in pulse length & $0.1 \mathrm{ps}$ \\
\hline Final Output wavelength & $355 \mathrm{~nm}$ \\
\hline Optional output wavelength & $532 \mathrm{~nm}$ \\
\hline Beam Quality @ 355 nm & TEM00; $\mathrm{M}^{2} \leq 1.5$ \\
\hline $\begin{array}{c}\text { Optimized for a required power } \\
\text { at 355 nm }\end{array}$ & $>5 \mathrm{~W}$ \\
\hline Average output power stability at & $<1 \% \mathrm{rms}$ \\
\hline 355 nm & $<1 \%$ rms \\
\hline Amplitude noise & Less than 3\% of the beam radius (1/e2 level) \\
\hline Centroid Position Stability & Less than $25 \mathrm{microradian}$ \\
\hline Pointing Stability & $\begin{array}{c}\text { Less than } 0.5 \% \text { of total UV energy within }+/- \\
100 \text { ps of laser pulse }\end{array}$ \\
\hline $\begin{array}{c}\text { Pre- and post-pulses and } \\
\text { pedestals, temporal halo }\end{array}$ &
\end{tabular}

Table 1 Laser Requirements

Other accelerator physics requirements dictate the remaining parameters, and their values come out of our beam dynamics studies. Amplitude fluctuations translate into electron bunch charge fluctuations. Stability of the pointing and centroid position translate into the stability of the electron bunch source position in the gun. Finally, the high repetition rate means that even small charge production at RF phases away from the electron bunch can be quite serious, and this accounts for the pre- and post-pulse contrast limits.

\section{$\underline{\text { Laser Description }}$}

A schematic diagram of the laser system is shown in Fig. 2, and the physical layout of the subsystems on the optical breadboard is displayed in Fig 3. The Seeder is a mode-locked $\mathrm{Nd}: \mathrm{YVO}_{4}$ oscillator, end-pumped with $25 \mathrm{~W}$ of $808 \mathrm{~nm}$ light, which is fiber-coupled in from diodes located in an off-board power supply. A semiconductor saturable absorber mirror (SESAM) is used for mode-locking. A White-cell multipass configuration is used to achieve the long path length required for our low, $9.38 \mathrm{MHz}$ repetition rate [1]. This is 


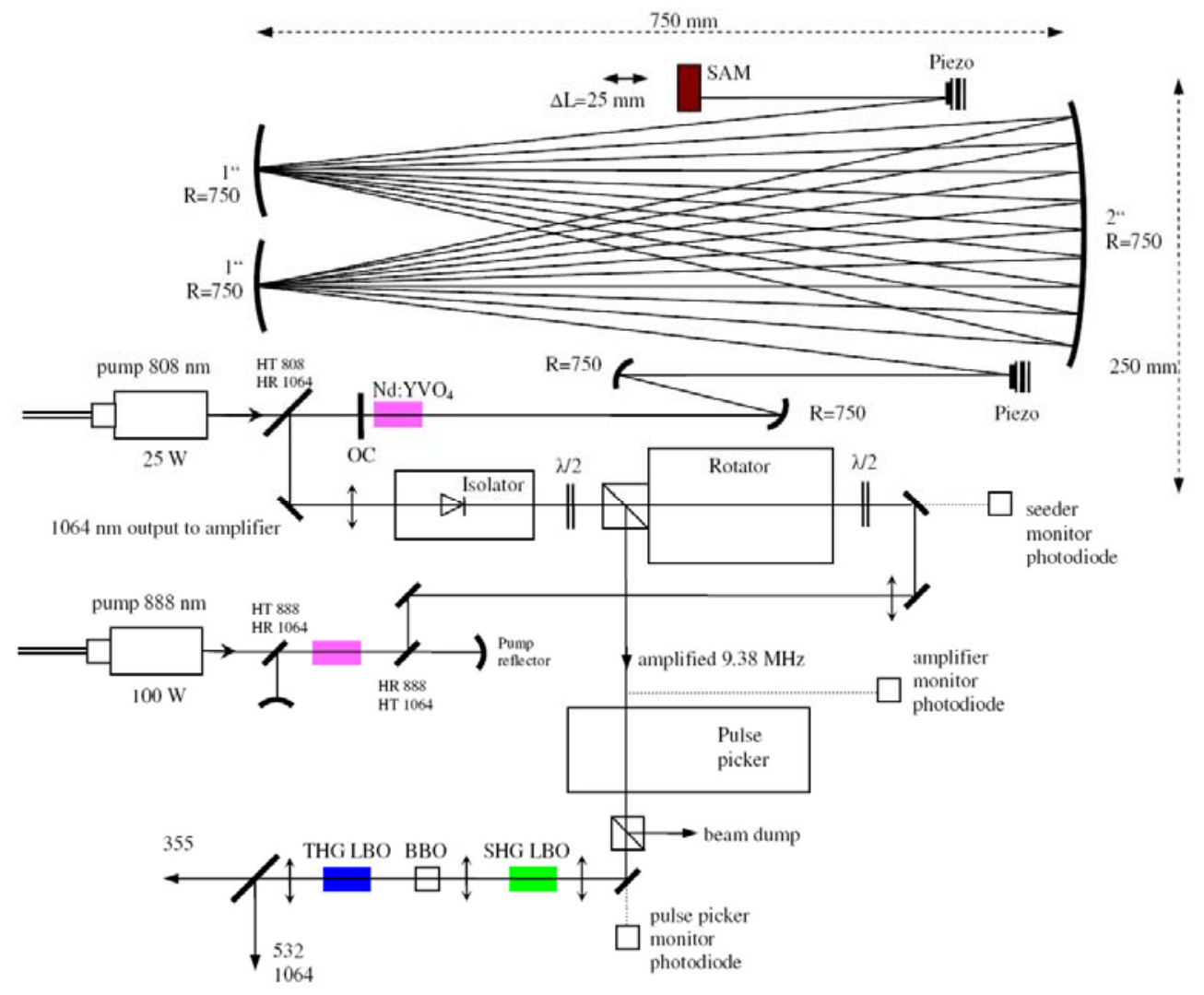

Figure 2 Functional schematic diagram of IDL Rapid Laser from Lumera GmbH. The ray fans at the top represent the multiply folded path in the Seeder. Following isolation and amplification, the $1064 \mathrm{~nm}$ pulses are selected (Pulse Picker) and frequency-doubled (SHG LBO). The doubled light is then mixed with the remaining IR to produce $355 \mathrm{~nm}$ (THG LBO)

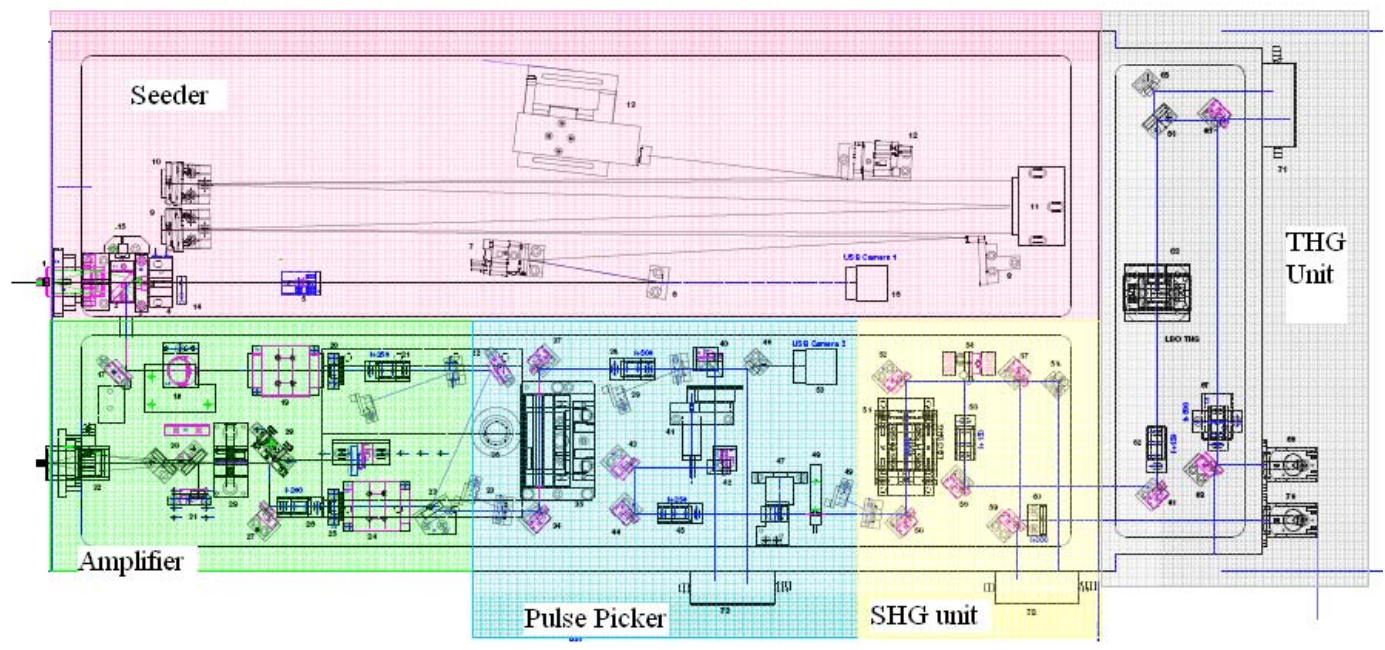

Figure 3 Physical layout of Lumera laser optical subsystems. Pump sources are located in a separate unit. 
a cavity folding technique which uses a cell comprised of three mirrors of identical curvature that repeatedly image the spot to the mirror surface, each time with a small displacement, so that the beam ultimately exits the cell after a large number of traversals (illustrated by the ray fans at the top of Fig 2).

The oscillator is followed by an isolation stage and a Nd:YVO $\mathrm{YV}_{4}$ power amplifier. The 100 watts of pump light for the amplifier is again brought in by fiber from off-board diodes. The $2.2 \mathrm{~W}, 1064 \mathrm{~nm}$ output of the oscillator is amplified to $20 \mathrm{~W}$ in the amplifier. A pulse picker follows, consisting of a BBO Pockels cell in conjunction with a polarizer. The rise time of the Pockels cell is $5 \mathrm{nsec}$, enabling it to select single pulses or groups of pulses at burst rates up to $1 \mathrm{kHz}$, with up to $90 \%$ duty cycle. Continuous operation at the full $9.38 \mathrm{MHz}$ is also possible. The desired pulse pattern can be simply set from the ERL control system through TTL signals.

Laser Pulse Reptition Frequency

Output Power

Synchronization timing jitter

Pulse Length (1064 nm)

Jitter in pulse length

Beam profile parameters, $355 \mathrm{~nm}$

Beam profile parameters, $532 \mathrm{~nm}$

Beam profile parameters, $1064 \mathrm{~nm}$

\section{$9.38 \mathrm{MHz}$}

$355 \mathrm{~nm}$

$532 \mathrm{~nm} \quad 6.5 \mathrm{~W}$

$1064 \mathrm{~nm} 20 \mathrm{~W}$

600 fsec $(10 \mathrm{~Hz}-1 \mathrm{MHz})$

10 psec

not measured

radius $\left(1 / \mathrm{e}^{\wedge} 2\right)$

ellipticity

$0.74 \times 0.64$

0.87

radius $\left(1 / \mathrm{e}^{\wedge} 2\right)$

ellipticity

$0.90 \times 0.78$

0.88

radius $\left(1 / \mathrm{e}^{\wedge} 2\right)$

ellipticity

M2X

$\mathrm{M} 2 \mathrm{Y}$

Average output power stability at $355 \mathrm{~nm}$

Amplitude noise

Centroid Position Stability

Pointing Stability

contrast (355 nm)
$0.72 \times 0.70$

0.98

1.15

1.1

$<1 \%$ rms

$<1 \%$ rms

Less than $3 \%$ of the beam radius ( $1 / \mathrm{e} 2$ level)

Less than 25 microradian

3.E-06

Table 2 Lumera laser performance summary

The selected optical pulses are then passed sequentially through the second harmonic generation (SHG) and third harmonic generation (THG) stages, producing $532 \mathrm{~nm}$ and $354.7 \mathrm{~nm}$ light respectively. Both harmonic stages use LBO as the nonlinear crystal, chosen for its high damage threshold and high conversion efficiency. In the SHG stage, noncritical phase matching is used to minimize impact on the mode quality. The conversion efficiencies are 50\% for the SHG and 35-40\% (of the total $532+1064 \mathrm{~nm}$ input power) for the THG. 


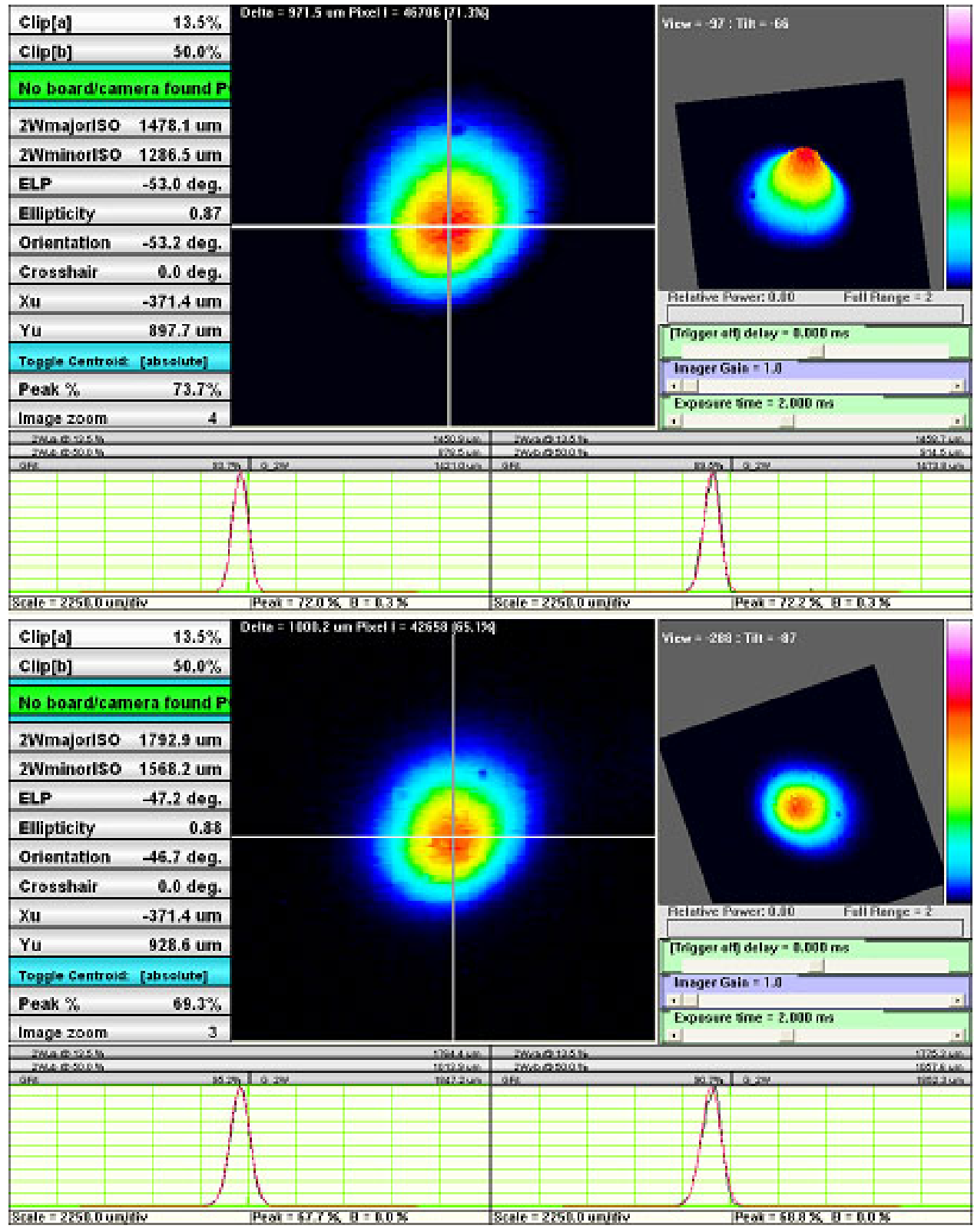

Figure 4 Laser beam profiles after installation. (top) $355 \mathrm{~nm}$, (bottom) $532 \mathrm{~nm}$

Performance of the laser as delivered is summarized in Table 2 and Figure 4. Some tests of beam power at all three wavelengths, spot profiles, pulse picking, and pulse contrast were done at BNL when the laser was delivered. Jitter, stability, pulse width, and other measurements were done and certified to meet specifications during design reviews at the manufucturer's facility, and will be repeated at BNL when the ERL's laser facility is operational in January 2010. Of some concern at this point is the deviation of the ellipticity of the beam from unity, as the spatial pulse shaping is quite sensitive to mode. However we do not expect this problem to be critical, as it is in principle possible to spatially filter the beam in the front end of the spatial shaping stage. 
a)

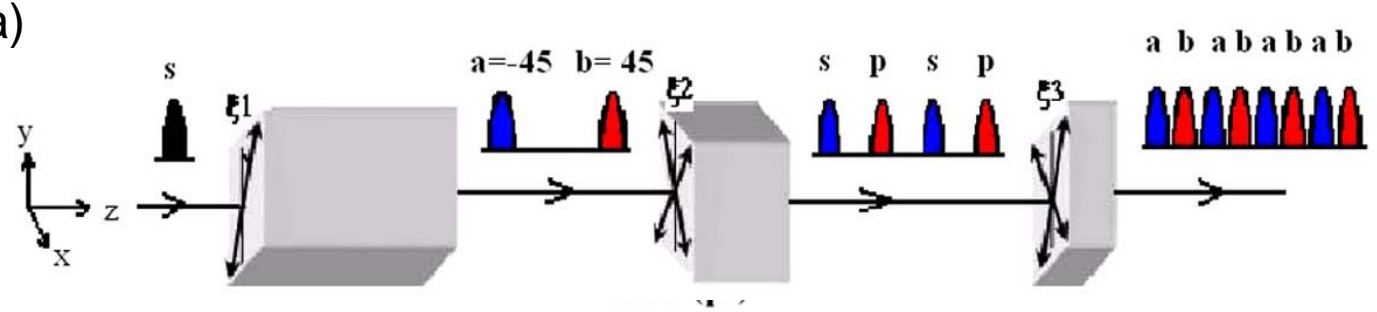

b)

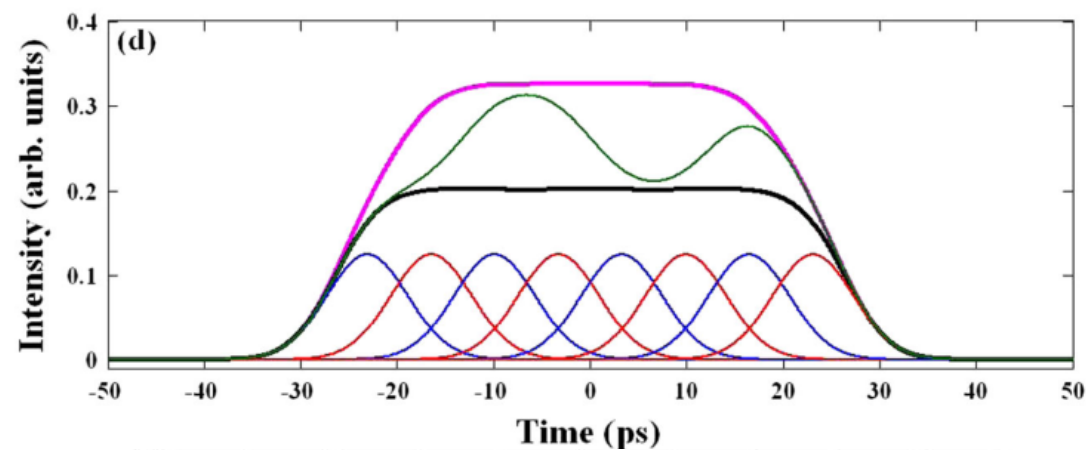

c)

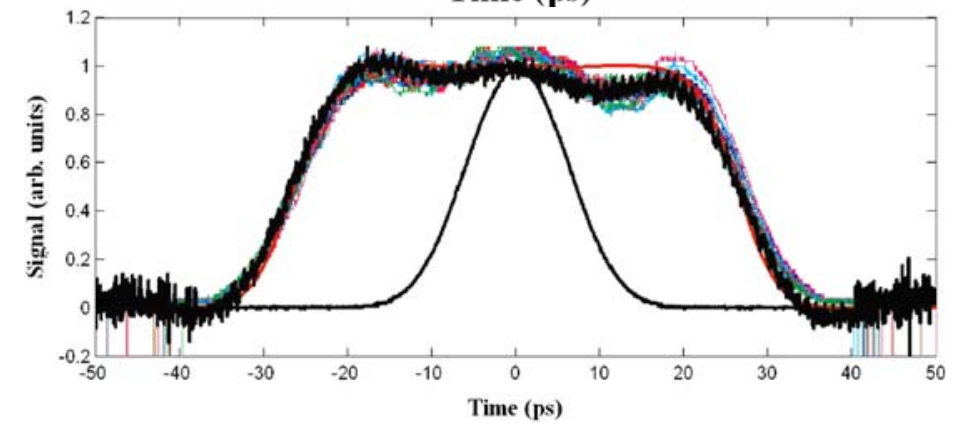

Figure 5 a) Birefringent crystal arrangement for pulse stacking, the fast axis of each crystal is rotated 45 degrees relative to the previous crystal. b) theoretical pulse shape given by summing a series of eight 10-psec FWHM Gaussian pulses with alternating orthongonal polarization (red and blue curves) separated in time by 6.5 psec, with static phases between neighboring pulses of 0 (magenta), $\pi / 2$ (black), and alternately 0 and $\pi / 2$ (green). c) experimental test (see text for details). (from Ref. [4], (C) 2008 American Physical Society)

\section{Temporal Shaping}

To limit emittance growth from space-charge effects, the optimal pulse at the photocathode is 50-120 picoseconds long, with a flat top, and rise and fall times of a few picoseconds. Mode-locked laser pulses have a sech ${ }^{2}$ temporal shape, which is very similar to a Gaussian shape, with slightly more intensity in the wings of the distribution. Our approach to producing flat-top pulses is to "stack" our picosecond pulses sequentially in time, with the polarizations of neighboring pulses being orthogonal in order to minimize interference effects. There are two approaches to doing this. One method is to use a number $\mathrm{N}$ of birefringent crystals to progressively duplicate and timeshift pulses such that the resulting series of $2^{\mathrm{N}}$ pulses overlap in such a way as to sum to a flat top pulse [2-4]. 
This technique is illustrated for an eight-pulse series in Fig. 5(a). The first crystal is oriented so that the fast axis makes a 45-degree angle with the laser polarization. The fast and slow polarization components traverse the crystal with different delays, so that at the output, there are two orthogonally polarized pulse replicas separated by a time that is determined by the crystal length, which we can define as $4 \Delta \tau$. These pulses are again each divided by a second crystal, half as long, oriented at 45 degrees to the first, which introduces a delay difference of $2 \Delta \tau$ between replicas, and finally these 4 pulses are each divided by a third crystal with delay difference $\Delta \tau$. The net effect is to produce a series containing eight pulse replicas of alternating orthogonal polarization, with a separation of $7 \Delta \tau$ from the first to the last.

If $\Delta \tau$ is chosen to be less than the width (FWHM) of the laser pulse, it is possible to approximate a flat-top pulse. Fig. 5(b) shows the simulated pulse shape obtained by summing 8 replicas of a 10 psec FWHM pulse, separated by $6.5 \mathrm{psec}$. Note that the method is very sensitive to interference effects between the pulses, exemplified here by the profound effect that varying the static phase between replicas has on the flatness, height, and width of the output pulse.

As part of an ongoing effort to evaluate the suitability of this method for use with our gun, we conducted tests of this technique with a laser similar to the ERL prototype laser. We used the frequency-doubled, $532 \mathrm{~nm}$ output of a Time-Bandwidth Cheetah-X, 81 $\mathrm{MHz} \mathrm{Nd:} \mathrm{YVO}_{4}$ laser[5]. The pulse width was 10 psec FWHM, and average power was $2.5 \mathrm{~W}$. Three $a$-cut yttrium orthovanadate $\left(\mathrm{YVO}_{4}\right)$ crystals of lengths 24,12 and $6 \mathrm{~mm}$ were used to create, from each pulse, 8 replica pulses of alternating orthogonal polarizations, separated by $6.5 \mathrm{psec}$. The resulting approximately rectangular pulse is measured with a scanning cross-correlator, employing second harmonic generation in a KDP crystal, using part of the original 10 psec pulse train in the reference arm (see left hand portion of Fig. 8). Further experimental details can be found in Ref. [4]

Static phase differences between replicas can arise from unequal path lengths traversed by the ordinary and extraordinary pulses, as a result of the tilt of the crystal plane. These phase differences can change over time due to thermal expansion and temperaturedependent birefringence of the crystals, particularly since the optical absorption in this case is significant ( $32 \%$ over all 3 crystals). Hence, the pulse shape can drift with time. Fig 5(c) shows this drift over a four hour time period. Cross correlations were taken every 10 minutes and are shown as the thin colored lines. The heavy black line is the deconvolved pulse shape, while the thin black line is the autocorrelation of the $10 \mathrm{psec}$ input pulse. The solid red line represents the theoretical pulse shape, assuming zero static phase in all replicas.

The deviation from flat top in the $53 \mathrm{psec}$ FWHM pulse is close to the $9 \%$ predicted by theory, and the variations in shape over time were observed in a standard laboratory environment and would likely be improved with careful temperature control of the crystals. There are, however, other challenges in extending this technique to the $355 \mathrm{~nm}$ pulse train to be used in the ERL prototype that must also be considered. The most suitable candidate for birefringent crystal to be used at this wavelength would be $\alpha-\mathrm{BBO}$. 
Considerable lengths of the crystal would be needed, and it is not clear that the optical homogeneity required can be obtained.

Another option is to do the pulse stacking interferometrically (see Fig. 6). This approach lacks the optical simplicity and robustness of the birefringent method, and is extremely sensitive to alignment. We have done some preliminary tests using the Cheetah-X laser and did find the alignment issues challenging. On the other hand, it is more versatile both in reaching longer pulse lengths, and in fine-tuning the pulse replica overlap, and involves traversing less material. It has been successfully implemented at Spring8 [6], so it seems that the engineering issues can be surmounted. A hybrid configuration mixing birefringent and interferometric stages is also possible and might offer a compromise solution that is reasonably versatile and stable.

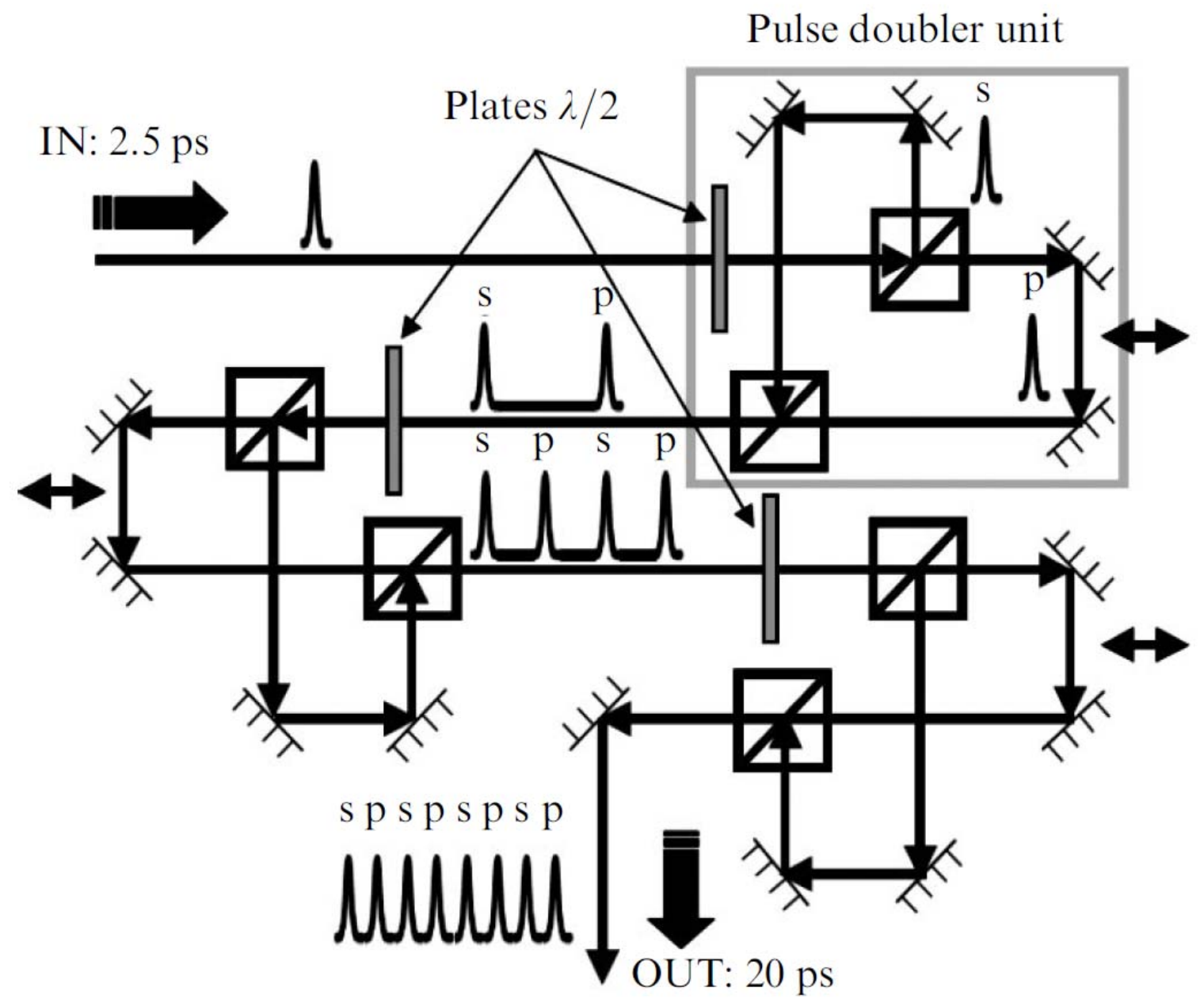

Figure 6 Optical Layout of an interferometric pulse stacker, reproduced from Ref [6]. The pulse doubler unit enclosed in the grey box is repeated 3 times to generate a series of 8 pulses of alternating orthogonal polarization. The $\lambda / 2$ plates rotate the polarization by 45 degrees before each stage. The cubes are polarizing beam splitters and the hatched surfaces represent mirrors. Displacing the mirror pairs indicated with arrows sets the pulse separation in each stage $\odot 2007$ Institute of Physics. 


\section{$\underline{\text { Spatial Shaping and Transport }}$}

It is equally important to spatially shape the pulse, in order to obtain, as nearly as possible, a flat-top transverse profile on the photocathode. Efficient spatial pulse shaping can be achieved with commercial devices, known as $\pi$-shapers, which are essentially telescopes fabricated with aspheric lenses so that the magnification is radially dependent[7]. A Gaussian input can then be transformed into a flat top, with deviations from flat top on the order of $10-15 \% \mathrm{rms}$ in commercial devices[8], with efficient use of nearly all of the pulse energy.

These devices are very sensitive to small deviations of the input pulse parameters from their design values, and to any degradation in mode quality. Also, in our application, we must make the spatial shaping work together with the temporal shaping, transport the shaped pulse over 9 meters and image it on the photocathode. In order to test in principle the entire optical design, we tested a commercial shaper [8], first in combination with a model of our transport line and then in combination with both the transport and the temporal shaping described in the previous section. As a full description may be found in Ref [4], we will give a brief description and summarize the results here.

(a)

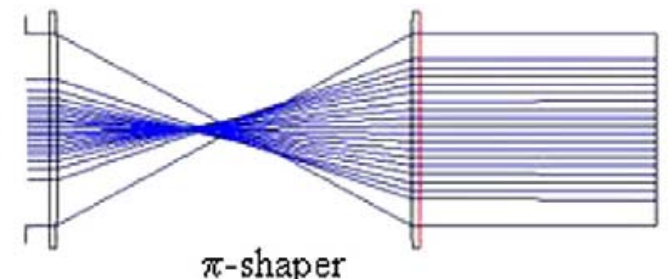

$\pi$-shaper

(b)

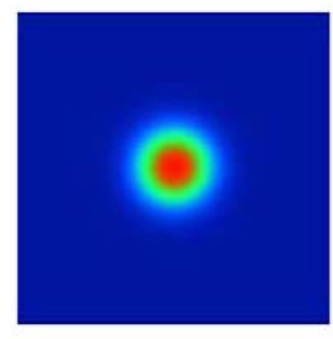

(c)

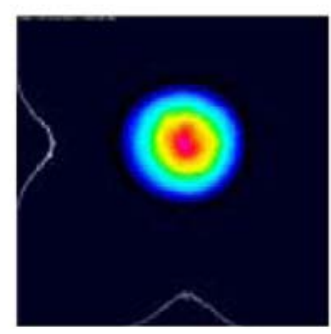

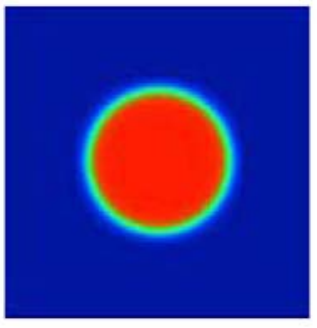

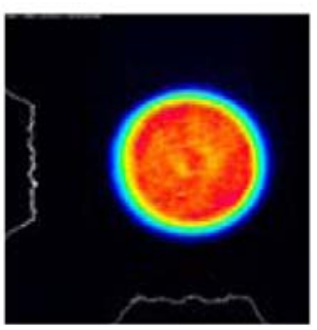

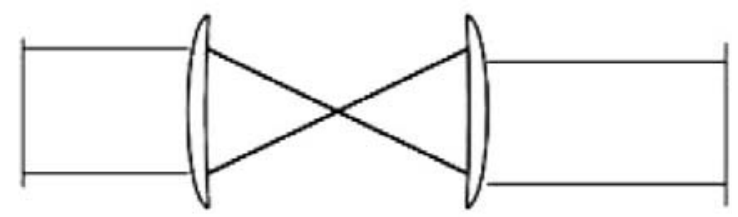

image-relay
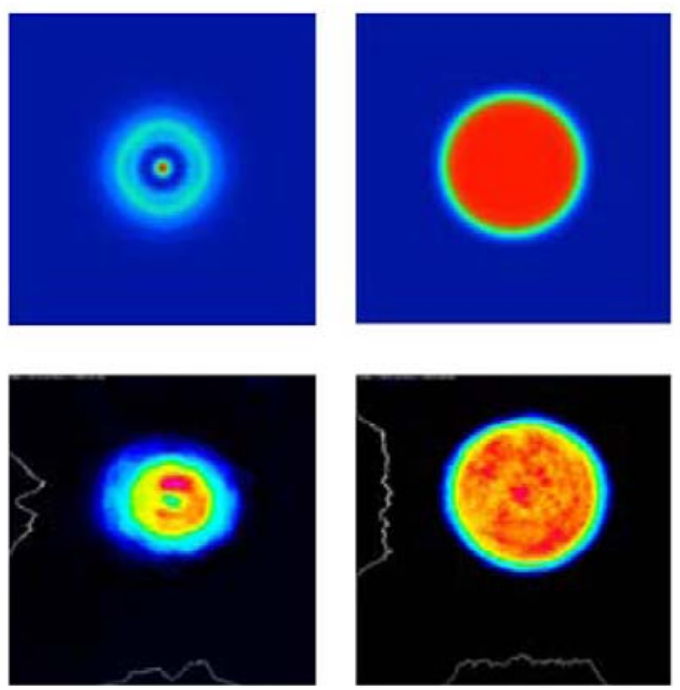

Figure 7 (a) Left: ZEMAX simulated ray diagram for the $\pi$-shaper (vertical scale expanded by 10X); right: schematic of the image-relay system. (b) Left to right: ZEMAX simulated beam profiles of the Gaussian input beam, output flattop beam at $10 \mathrm{~cm}$, at $9 \mathrm{~m}$ without image-relay lens, and at $9 \mathrm{~m}$ with image-relay lens. (c) Corresponding measured profiles. (from Ref. [4], (C) 2008 American Physical Society) 


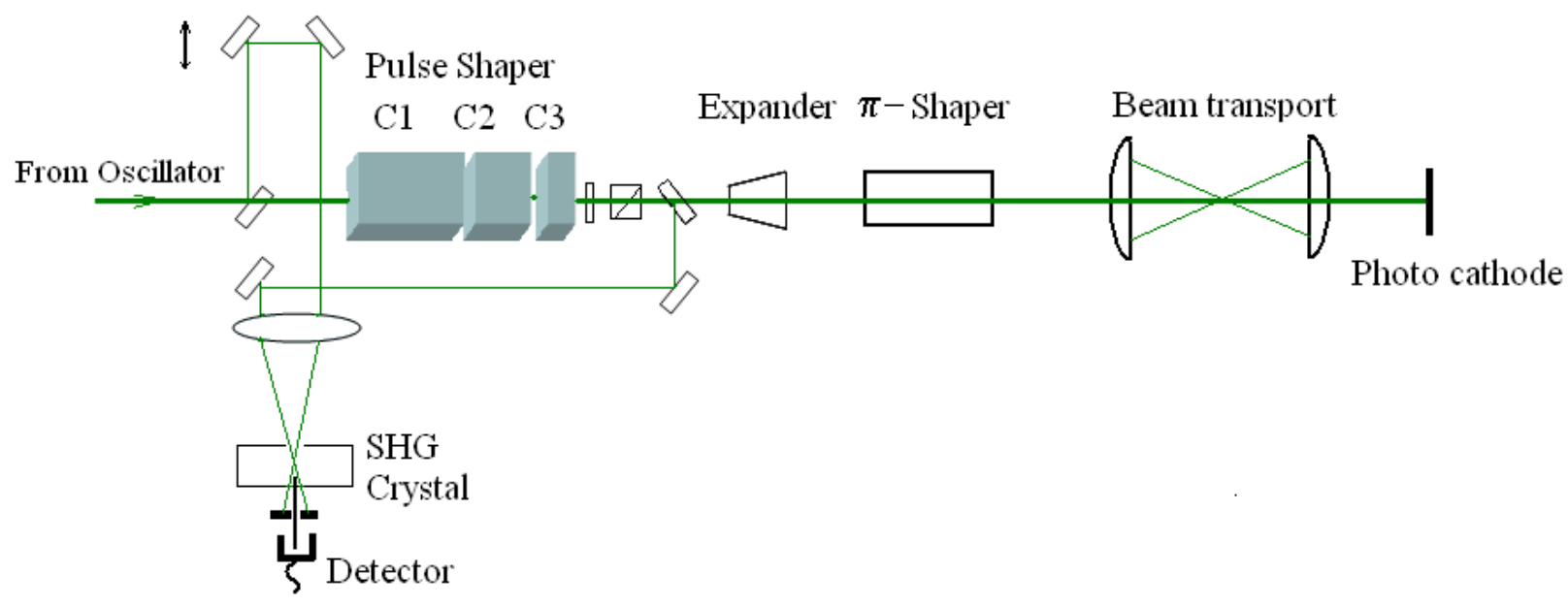

Figure 8. Experimental configuration for testing temporal shaping, spatial shaping and beam transport. The unshaped pulse is used as reference in the cross-correlator in the left of the figure.

We found the $\pi$-shaper performance in good agreement with our ZEMAX simulations, as shown in Fig. 7. We were able to transport the flat top over a 9 meter transport line, which consisted of a unit magnification Keplerian telescope containing 2 plano-convex lenses of focal length $\mathrm{f}=2.25 \mathrm{~m}$, separated by 4.5 meters, and multiple turning mirrors. The measured deviations from flat top suffered some deterioration over the transport, increasing from $7 \% \mathrm{rms}$ before the transport line to $10 \% \mathrm{rms}$ at the image plane. The depth of focus of the flat top, or the distance over which no diffraction rings appeared in the beam profile, was approximately $50 \mathrm{~cm}$, and decreased to $\sim 30 \mathrm{~cm}$ when we increased the magnification of the transport relay from 1 to 2 . The measured transmission was $92 \%$, and tolerance to tilt and decenter $( \pm 9 \mathrm{mrad}$ and $\pm 0.38 \mathrm{~mm})$ are much larger than required by our laser's position and pointing stability.

The optical layout of the full test is shown in Fig 8 . The temporal shaping is done first, as the limited depth of focus of the $\pi$-shaper affects the cross-correlation measurement. A beam expander is used to match the beam into the $\pi$-shaper. We also tried expanding the beam prior to the temporal shaping stage, and found that increasing the beam diameter there degraded the transverse profile, which can seen in the appearance of interference fringes in Fig 9(a), compared to 9(b). Potential sources of this include small walk-off effects in the crystals, piezoelectric effects, and optical rotatory dispersion; In any case, a beam size limitation may impose a pulse energy limitation on the birefringent method of pulse stacking. The transported fully-shaped beam is shown in Fig 9(c), with a perspective representation of the "beer can" form of the pulse in Fig 9(d). 

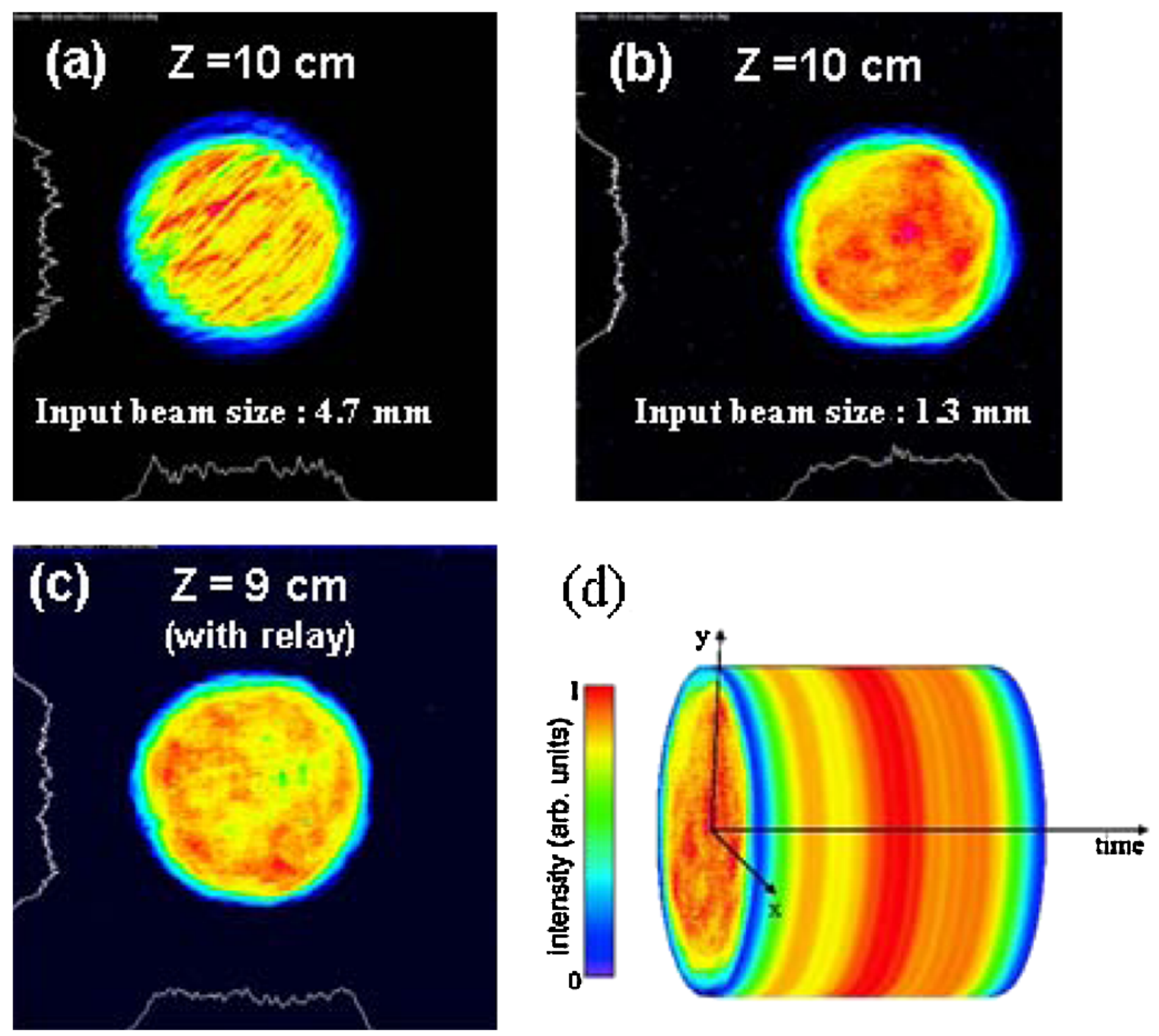

Figure 9 Spatial beam profiles. (a) profile $10 \mathrm{~cm}$ after the $\pi$-shaper, when beam expansion is done before temporal shaping instead of after; not the appearance of interference fringes. (b) profile $10 \mathrm{~cm}$ after the p-shaper; (c) profile (b) after $9 \mathrm{~m}$ optical relay. (d) reconstructed 3-D beam envelope of the spatiotemporal shaped beer-can light pulse profile. (from Ref. [4], (c) 2008 American Physical Society)

\section{$\underline{\text { Diagnostics and Control }}$}

A more detailed description of the components and methods of the full control system can be found in the Controls chapter, but it is worthwhile to highlight here what measurements and inputs are essential for the optical system. The system overview in Fig. 1 represents this schematically. Control of the laser itself - RF reference, shuttering, power, and pulse pattern - is done through signals from the ERL control system and its interface with the laser's native computer control system. The laser provides an error signal from its own phase-locked loop control of its oscillator repetition rate, but we also make an external jitter measurement at the laser output, using an RF signal derived from a photodetector looking at the laser, mixed with the RF reference. This also gives us the capability to look for jitter using a signal derived from a detector placed at the end of the transport line, and so detect overall path length fluctuations. Pulse amplitude stability and 
timing stability relative to the controller-requested pulse pattern will also be verified at the laser output.

The temporal shape of the pulse must be measured both at the laser and following the temporal shaping stage. A cross correlator has been developed for this, and is being integrated into the control system. It currently measures the $532 \mathrm{~nm}$ alternate output of the Lumera laser, but will be modified to measure the $355 \mathrm{~nm}$ directly. In addition we are working on developing a sub-picosecond pulse from the alternate output of the laser, by using fiber pulse compression, to provide a higher-resolution time diagnostic. This is motivated by a concern that interference effects between replicas in pulse stacking will show up at shorter time scales than can be seen with 8-10 psec resolution of the current method. The controller input at the temporal shaping stage is denoted in Fig 1 with a dashed line and a question mark to indicate that control will almost certainly not be realtime, and its extent is still not known. As noted above, birefringent pulse stacking fixes the pulse delays with the length of the crystal cut. While interferometric methods offer delay tunability in principle, their alignment sensitivity means that significant adjustability will likely be between experimental runs rather than dynamic.

Power measurements and beam profile measurements will be made in as many locations as possible, at least after each of the main stages shown in Fig. 1. These measurements are easy to integrate; they can be as simple as photodiodes or inexpensive cameras looking at beam pickoffs or mirror leakage, and can be used to rapidly locate the source of potential problems during an experimental run.

The 'monument' consists of an image plane conjugate to the location of the photocathode, obtained by picking off a small amount of light near the beam entrance to the gun chamber and propagating it a distance equal to the separation of the pickoff and the photocathode. A large format, high resolution CCD camera is placed in this plane, to give an accurate picture of the beam location, movement, and profile on the photocathode.

\section{$\underline{\text { Summary }}$}

The ERL project requires an extensive laser pulse generation and control system for reliable operation. A great deal has been done towards developing this system and, while much work remains, it seems tractable within the allotted time frame and does not appear to present any critical impediments to the project. The laser itself must be more fully characterized on site, but measurements made during extensive design reviews indicate that any problems would not be ones of essential design and would be reparable. Open questions remain in the optimal design of the pulse shaping, as discussed above, but work done to date makes us confident that they are indeed issues of the solution's optimization, not its existence. Transport of the shaped beam has also been demonstrated in principle. This leaves, of course, the task of demonstrating it stably with this system in the ERL environment, but this does not seem especially problematic. The system on the whole seems compact, robust and flexible. 


\section{$\underline{\text { References }}$}

1. Chen, J., R. Knappe, and W. Viöl, Passively mode-locked Nd:YVO4 multipass resonator with low repetition rate. Laser Physics Letters, 2008. 5(6): p. 425-428.

2. Bates, H.E., R.R. Alfano, and N. Schiller, Picosecond pulse stacking in calcite. Appl. Opt., 1979. 18(7): p. 947-949.

3. Zhou, S., et al., Efficient temporal shaping of ultrashort pulses with birefringent crystals. Appl. Opt., 2007. 46(35): p. 8488-8492.

4. Sharma, A.K., T. Tsang, and T. Rao, Theoretical and experimental study of passive spatiotemporal shaping of picosecond laser pulses. Physical Review Special Topics - Accelerators and Beams, 2009. 12(Copyright (C) 2009 The American Physical Society): p. 033501.

5. Time-Bandwidth Products, Halle Zeppelin 1. OG, Technoparkstrasse 1, 8005 Zurich, Switzerland, www.tbwp.com.

6. Tomizawa, H., et al., Development of a yearlong maintenance-free terawatt Ti:Sapphire laser system with a 3D UV-pulse shaping system for THG. Quantum Electronics, 2007. 37(8): p. 697-705.

7. Hoffnagle, J.A. and C.M. Jefferson, Design and Performance of a Refractive Optical System that Converts a Gaussian to a Flattop Beam. Appl. Opt., 2000. 39(30): p. 5488-5499.

8. Newport Corporation, 1791 Deere Avenue, Irvine, CA 92606; Model GBS-AR14; http://www.newport.com/Refractive-Beam-Shapers/315393/1033/catalog.aspx. 